\title{
Direct real-time PCR examination for Mycobacterium tuberculosis in respiratory samples can be cost effective
}

\author{
Bryan Joseph Renton ${ }^{1}$, Patricia Denise Morrell ${ }^{2}$, Richard Peter Davidson Cooke ${ }^{2}$, Peter David \\ Owen Davies ${ }^{1}$
}

${ }^{1}$ Department of Respiratory Medicine, University Hospital Aintree, Liverpool; Correspondence author: bjrenton@doctors.net.uk

${ }^{2}$ Department of Clinical Microbiology, University Hospital Aintree, Liverpool

Received 5 June 2009; revised 15 July 2009; accepted 18 July 2009.

\begin{abstract}
Aim: To assess whether the use of direct realtime polymerase chain reaction (PCR) on smear-positive sputa can be cost-effective, by speciating mycobacteria earlier than current methods and thereby preventing unnecessary screening tests as part of the contact tracing process.

Methods: A retrospective study of all patients with smear-positive sputa in a Liverpool teaching hospital between 2004 and 2007. All the PCRs performed on these patients were reviewed and compared them with their mycobacterial culture results. Unit costs for PCR, chest X-ray (CXR), tuberculin skin test (TST), interferon-gamma (IFN-y) and medical/nursing time were conservatively estimated at $£ 50, £ 11$, $£ 10, £ 40$ and $£ 30$ respectively. The total PCR costs were compared with the costs of unnecessary follow up of patients, negative for Mycobacterium tuberculosis (MTB) by PCR, subsequently confirmed to be MTB culture negative.
\end{abstract}

Results: 203 smear-positive patients underwent direct PCR testing. 126 (62\%) patients grew Mycobacterium tuberculosis (MTB), 74 (37\%) had environmental mycobacterial infection (EMI) and $3(1 \%)$ were culture negative. Of the 126 patients' culture positive MTB patients, 123 were PCR positive and 3 PCR negative. Of the 77 patients that were culture negative for MTB, 75 were PCR negative and 2 PCR positive The sensitivity, specificity, positive and negative predictive values for direct PCR versus MTB culture were $98 \%, 96 \%, 98 \%$ and $97 \%$ respectively. Total costs of all PCRs performed amounted to $£ 10,150$. The cost of contact procedures for PCR-negative and MTB culturenegative index cases was estimated at $£ 19,650$. This equated to a total saving of $£ 9,500$ in con- tact tracing costs.

Conclusions: Direct PCR examination testing of smear-positive patients can be cost-effective in areas where there is a high incidence of EMI.

Keywords: Tuberculosis; PCR and Cost Effective

\section{INTRODUCTION}

Current national guidelines for diagnosing active pulmonary tuberculosis (TB) recommends performing a posterior-anterior chest X-ray (CXR) and obtaining at least 3 sputum samples (with one early morning sample), which are then sent for TB microscopy and culture. [1] These sputum smears are initially stained for acid-fast bacilli (AFB), but as Mycobacterium tuberculosis (MTB) and environmental mycobacteria have a similar microscopic appearance, it is not possible to differentiate between them at this stage. Mycobacterial culture and subsequent speciation is therefore required but is often not available until several weeks after specimen collection.

In patients suspected of active TB, which is usually based on a smear-positive sputum or bronchoalveolar lavage (BAL), a contact tracing process is initiated early to prevent potential further spread. Screening is usually offered to household and other close contacts. Typically, most contacts will have a chest X-ray and a tuberculin skin test (TST), with a significant proportion of contacts also going on to have an interferon-gamma (IFN- $\gamma$ ) test. However, in areas where there is a high incidence of environmental mycobacterial infection (EMI), this can result in a large proportion of contacts being unnecessarily screened. Indeed, in the study carried out by Corless et al., 51\% of smear positive patients did not have TB. This resulted in $31 \%$ of contacts of patients being screened who either cultured environmental organisms, or had negative cultures. The median number of contacts traced per index case was 11 for patients culturing MTB and 4 for patients culturing environmental organisms or with negative cultures. This report highlighted the cur- 
rent inefficiencies that exist in the contact tracing procedures. The authors felt that there are clear grounds for using rapid tests to identify and type mycobacteria more quickly than current solid or liquid media methods; thereby avoiding extra unnecessary screening costs. [2] This would certainly seem to be applicable to areas with a high incidence of lung disease caused by EMI, but unfortunately there still remains no published national database on this topic. However, a further review of mycobacterial isolates cultured from respiratory samples in Merseyside 2000 - 2008 has again confirmed the high proportion (approximately 50\%) of smear positive sputum that yield environmental mycobacteria.

Polymerase chain reaction (PCR) allows for more rapid identification of $\mathrm{MTB}$, but is not routinely recommended, principally due to cost implications. Current national guidance advises its use only if rapid confirmation of a TB diagnosis in a sputum smear-positive person would alter their care (e.g. exclusion of non-tuberculosis mycobacteria in immunocompromised patients), or before conducting a large contact tracing initiative (e.g. in a school or hospital). [3] In this study, we aimed to assess whether the use of direct PCR on smear-positive sputa can be cost-effective, by speciating mycobacteria earlier than current methods, and therefore preventing unnecessary screening tests as part of the contact tracing process.

\section{MATERIALS AND METHODS}

This retrospective study was carried out at a large teaching hospital in Liverpool (University Hospital Aintree). All AFB smear-positive respiratory specimens underwent direct PCR testing for MTB and Mycobacterium avium-intracellulare complex. PCR testing on smearpositive sputa has been routine practice in this hospital for the last 4 years, initially on a weekly basis, and due to increasing clinical demand, is now performed twiceweekly. On receiving the smear-positive sample, the average time to issue a PCR result is between $48-72$ hours. Once this result is available, the responsible clinician is notified immediately.

All the direct PCRs performed on patients with smear-positive sputa between 2004 and 2007 were reviewed and compared with their mycobacterial culture results.

A real-time PCR assay (Real ArtTM Mycobac. Diff. LC PCR kit, artus Biotech USA) was used for the detection of MTB complex (human MTB, M. bovis, M. africanum, M. microti, M. cannetti) and Mycobacterium avium-intracellulare complex. A region of the mycobacterial 16S DNA, conserved in all members of MTB complex, was amplified and detected by the specific melting point temperature of the flurogenic probe used [4].

The assay system contains, in one master mix, all reagents and enzymes for the specific amplification and detection of a 163 base pair region of the MTB genome. The kit also provides a dilution series of external positive MTB controls for precise quantification of the MTB complex load. PCR inhibitors are also to be detected to prevent the generation of false negative results. An internal control, co-amplified with the specific target DNA in the same capillary, is therefore included in the assay kit. This internal control does not influence the sensitivity or specificity of the MTB PCR. If the internal control is added to the sample before the nucleic acid isolation process, it can also be used to control the efficiency of the DNA extraction procedure.

Unit costs for PCR, CXR, TST and IFN- $\gamma$ were estimated at $£ 50, £ 11, £ 10$ and $£ 40$ respectively. We also conservatively estimated a further minimum cost of $£ 30$ per patient, which incorporated district nurse, TB control nurse and medical time. We aimed to compare the total PCR costs with the costs of unnecessary follow up of PCR negative patients, who subsequently proven to be MTB culture negative.

The cost of contact procedures for PCR-negative and MTB-culture negative index cases was based on the assumption that there were 4 contacts traced per index case for patients with EMI. [2]

\section{RESULTS}

A total of 203 smear-positive patients underwent direct PCR testing. $126(62 \%)$ patients grew MTB, 74 (37\%) had EMI and $3(1 \%)$ were culture negative. Of the 126 patients that were culture positive for MTB, 123 were PCR positive and $3 \mathrm{PCR}$ negative. Of the 77 patients that were culture negative for MTB, 75 were PCR negative and 2 PCR positive (Table 1). The sensitivity, specificity, positive and negative predictive values for direct PCR versus MTB culture were 98\%, 96\%, 98\% and 97\% respectively.

Table 1. Comparison of PCR and culture results for 203, AFB smear-positive sputa.

\begin{tabular}{|c|c|c|c|c|}
\hline & \multicolumn{4}{|c|}{ MTB Culture } \\
\hline & & + & - & \\
\hline & + & 123 & 2 & 125 \\
\hline \multirow[t]{2}{*}{$\begin{array}{l}\text { MTB } \\
\text { PCR }\end{array}$} & - & 3 & 75 & 78 \\
\hline & & 126 & 77 & 203 \\
\hline $\begin{array}{l}\text { MTB: } \\
\text { PCR: P } \\
\text { *Sensiti } \\
\text { Specific } \\
\text { Positive } \\
\text { Negativ }\end{array}$ & $\begin{array}{l}\text { bacte } \\
\text { eras } \\
98 \% \\
96 \% \\
\text { dicti } \\
\text { edict }\end{array}$ & $\begin{array}{l}\text { berculo } \\
\text { reaction } \\
\text { e: } \mathbf{9 8 \%} \\
\text { le: } 97 \%\end{array}$ & & \\
\hline
\end{tabular}


Table 2. Breakdown of estimated costs for PCR tests and contact tracing procedures for PCR / M. tuberculosis culture negative index cases.

\begin{tabular}{|c|c|c|c|c|c|c|}
\hline TST & CXR & INF- $\gamma$ test & Medical/nursing & $\begin{array}{c}\text { Contact } \\
\text { tracing }\end{array}$ & PCR & Savings \\
\hline$£ 3,000$ & $£ 1,650$ & $£ 6,000$ & $£ 9,000$ & $£ 19,650$ & $£ 10,150$ & $£ 9,500$ \\
\hline $\begin{array}{l}\text { TST : Tube } \\
\text { CXR : Che } \\
\text { INF-y : Int } \\
\text { PCR : Poly }\end{array}$ & $\begin{array}{l}\text { n skin tes } \\
\text { ay } \\
\text { on-gamm } \\
\text { se chain }\end{array}$ & & & & & \\
\hline
\end{tabular}

The following costs per index case was identified:

Number of PCR-negative/MTB culture-negative cases (i.e. unnecessarily screened) $=75$

Number of contact cases per index case with EMI $=4$

Total cost for TST $=75 \times 4 \times £ 10=£ 3,000$

Total cost for INF- $\gamma$ (approximately 50\% of contacts go on to get this) $=75 \times 2 \times £ 40=£ 6,000$

Total cost for CXR (at least $50 \%$ of contacts require this) $=75 \times 2 \times £ 11=£ 1,650$

Total cost for medical/nursing time $=75 \times 4 \times £ 30=$ $£ 9,000$

Total costs of all PCRs performed amounted to $£ 10,150$ ( $£ 50 \times 203)$. The cost of contact procedures for PCR-negative and MTB culture-negative index cases was estimated at $£ 19,650$. This equated to a total saving of $£ 9,500$ in contact tracing costs (Table 2).

\section{DISCUSSION}

Previous studies looking at the cost effectiveness of PCR have focused primarily on the savings due to averted isolation, drug treatment, in-patient beds saved and further investigations. [5-7] To our knowledge, there have been no studies that have specifically looked at the cost-effectiveness of PCR in avoiding unnecessary contact tracing costs. We estimated that direct PCR use in this population of patients with smear-positive sputa, resulted in an overall net saving of $£ 9,500$ in contact tracing costs. Whilst this seems a relatively modest amount over a 3 -year time period, it is nevertheless, a saving, and justifies the expenditure on PCR $(£ 10,150)$. We also should not underestimate the other benefits that an early diagnosis will have. In our study, over a third of patients who had smear-positive sputa did not have MTB, which is in keeping with previous data in this area. [2] Currently, all smear-positive patients should be commenced on anti-tuberculosis treatment. In a population such as ours, this will result in a significant number of patients being incorrectly treated. Also, there are significant adverse effects that are associated with anti-TB therapy, [8] so these decisions should not be taken lightly. Prompt diagnosis using direct PCR may therefore also save on unnecessary treatment costs and adverse events which were not included in the costing model. Furthermore, from a psychological perspective, an early diagnosis will save a lot of patients' close contacts going through the stress of being subjected to a number of unnecessary tests to establish that they don't have MTB.

Whilst our study showed a saving in contact tracing costs of $£ 9,550$, this is likely to be a very conservative estimate. Once a person has been diagnosed with active $\mathrm{TB}$, the diagnosing physician should inform relevant colleagues so that the need for contact tracing can be assessed without delay. [1] This contact tracing process usually begins with a TB health visitor interviewing the patient. Screening is then offered to household and any other close contacts, which usually involves a visit to the TB screening clinic. After a TST, contacts will need a further visit to have the result read and if testing is inconclusive, they should be referred to a TB specialist. [1] All this equates to a significant amount of medical, health visitor and nursing time and it may well be that $£ 30$ per contact is a gross underestimate. Without a diary analysis of this time spent screening contacts, it was however, difficult to formally assess this. Overall, our estimate of $£ 9,550$ saved in contact tracing costs shows that PCR virtually pays for itself.

Microscopically, 5,000-10,000 AFB ml ${ }^{-1}$ must be present for smear-positivity, compared to 10-100 AFB ml ${ }^{-1}$ that are required for culture positivity. Therefore, a smear-positive respiratory sample signifies a high bacterial load and an increased risk of infectivity. In a mini review of available nucleic acid amplification tests (NAAT) for the detection of MTB in clinical specimens (21 studies), the mean sensitivity in smear-positive specimens was $94.5 \%$. [9] The positive-predictive value and sensitivity of the PCR in our study was $98 \%$, which is in-keeping with previous data on this particular realtime PCR assay (100\% sensitivity). [4] In view of this, and the fact that PCR is expensive, we feel that performing PCR on only one of the three smear positive specimens should be enough to aid diagnosis and decide which patients' contacts do and do not need to be screened. In contrast, NAAT sensitivity on smear-negative specimens was much lower, at $69.3 \%$. [10] However, as smear-negativity signifies a low bacterial load, these patients are likely to have a low risk of infectivity, so 
contact tracing becomes less of an urgent issue. As rapid liquid culture takes approximately 8-10 days in our hospital, we therefore felt that routine use of PCR in this group of patients would be unlikely to be cost-effective.

In a recent article by Taegtmeyer et al., the authors found that PCR had a direct impact on clinical management in one third of smear-positive patients in whom it was used. This included patients for whom treatment was changed and situations in which contact tracing exercises were either commenced or stopped. PCR significantly reduced the time to identification of the mycobacteria and detection or exclusion of rifampicin resistance, which allowed prompt alterations in management when needed. They concluded that there would be additional clinical benefit from PCR being applied to all smear-positive specimens in low prevalence settings. [10] We would concur with this, and believe that our study illustrates that direct PCR testing of all smear-positive patients can also be cost-effective.

In conclusion, although current national guidance on direct PCR recommends its use principally to confirm true MTB before a large contact tracing exercise is undertaken, our results suggest that it may have a useful and cost-effective role in routine clinical practice on smear-positive respiratory samples, particularly in areas with a high incidence of EMI. Whilst there are reports suggesting that the incidence of EMI has increased over the past few decades, this observation has not been conclusively established due to the lack of a comprehensive surveillance system. [11] This data would be particularly helpful in deciding which areas in the UK may benefit from direct PCR testing on all smear-positive respiratory specimens. Such an approach should be reflected in the National Institute of Clinical Excellence (NICE) guidelines when they are next revised.

\section{REFERENCES}

[1] CG33 Tuberculosis - National Institute of Clinical Excellent (NICE) guidelines. March 2006.

[2] Corless, J.A., Stockton, P.A., Davies, P.D. (2000) Myco- bacterial culture results of smear-positive patients with suspected pulmonary tuberculosis in Britain. Eur Respir $J$, 16: 976-9.

[3] National Collaborating Centre for Chronic Conditions: clinical diagnosis and management of tuberculosis and measures for its prevention and control. (2006). London, Royal College of Physicians.

[4] Cramer, S.O., Matinmehr, F, Hillemann, D. (2004) Fast and sensitive detection of the Mycobacterium tuberculosis complex by real-time PCR, Clinical Microbiology and Infection, 10(3), 418.

[5] Drobniewski, F.A., Watterson, S.A., Wilson, S.M., Harris, G.A. (2000) A clinical, microbiological and economic analysis of a national service for the rapid molecular diagnosis of tuberculosis and rifampicin resistance in Mycobacterium tuberculosis. J Med Microbiol, 49:271-8.

[6] Dowdy, D.W., Maters, A., Parrish, N., Beyrer, C., Dorman, S.E. (2003) Cost-effectiveness analysis of the Gen-Probe amplified Mycobacterium tuberculosis direct test as used routinely on smear-positive respiratory specimens. J Clin Microbiol, 41(3), 948-953.

[7] van Cleeff, M., Kivihya-Ndugga, L., Githui, W., Ng'ang'a L., Kibuga, D., Odhiambo, J., et al. (2005) Cost-effectiveness of polymerase chain reaction versus Ziehl-Neelsen smear microscopy for diagnosis of tuberculosis in Kenya. Int J Tuberc Lung Dis, 9:877-83.

[8] Zalekis, R. (2005) Side-effects of Tuberculosis treatment. Breathe, 2(1), 69-73.

[9] Piersimoni, C. and Scarparo, C. (2003) Minreview-Relevance of commercial amplification methods for direct detection of Mycobacterium tuberculosis complex in clinical samples. J Clin Microbiol, 41(12), 53555365 .

[10] Taegtmeyer, M., Beeching, N.J., Scott, J., Seddon, K., Jamieson, S., Squire, S.B., Mwandumba, H.C., Miller, A.R.O., Davies, P.D.O., Parry, C.M. ( 2007) The Clinical impact of nucleic acid amplification tests on the diagnosis and management of tuberculosis in a British hospital. Thorax Online.

[11] Griffith, D.E., Aksamit, T., Barbara, A., et al. (2007) American thoracic society guidelines: Diagnosis, treatment and prevention of nontuberculous mycobacterial diseases. Am J Resp and Crit Care Med, 175, 367-417. 